\title{
Research and Development of Automatic Width Measurement and Alignment Equipment for Cold Rolled Aluminum Strip Based on Machine Vision
}

\author{
Shan LIAN ${ }^{1}$, Lishan ZHANG, Sinuo XU, Hongfei LIU \\ School of Mechanical Engineering University of Science and Technology Beijing, \\ Beijing 100083, China
}

\begin{abstract}
Aiming at the inevitably offset phenomenon in the cold rolling process of aluminum strip, this paper introduces the automatic width measurement and centering scheme based on machine vision. With the Raspberry Pi as the main control unit, each frame of picture is processed through median filtering and Canny edge detection algorithm to obtain the edge pixel coordinates on both sides of the aluminum sheet, which are then converted to the world coordinate system to obtain its actual position. Calculate the width of the aluminum sheet via Ethernet communication to the main control computer; at the same time, calculate the offset of the aluminum sheet relative to the rolling line, and use the PID algorithm to control the hydraulic servo system of the uncoiling reducer to achieve closed-loop centering control. The actual measurement shows that this scheme effectively replaces the tedious operation steps of the console, and has good stability and performance.
\end{abstract}

Keywords. Cold reducing machine, machine vision, position servo system, edge detection, centering control.

\section{Introduction}

In the cold-rolling process of aluminum strip, due to the defects of the incoming strip and some reasons of the production equipment, the aluminum sheet will inevitably show deviation in the rewinding process [1-2]. In order to ensure the processing quality of the strip, it is necessary to detect and adjust the position of the aluminum strip during the cold-rolling process. The commonly used detection methods are inductive detection method, infrared detection method, photoelectric detection method and ultrasonic detection method. Inductance detection method is the use of the principle of inductance. According to the induced electromotive force generated by different positions of the strip, the edge position of the strip can be determined. Infrared detection method uses infrared light emitting/receiving diode through the strip to block the infrared signal and determine the edge position of aluminum sheet. The above two detection methods can adapt to harsh working conditions, without manual maintenance,

\footnotetext{
${ }^{1}$ Corresponding Author, Shan LIAN, School of Mechanical Engineering University of Science and Technology Beijing, Beijing 100083, China; Email: 1726372000@qq.com.
} 
both have a long operating life, but have a low resolution. They are both only suitable for strong magnetic conductive materials, and different materials have a great impact on the detection accuracy. Photoelectric detection method uses the shading effect of different positions of the strip to generate electrical signals, to get the edge position of the aluminum strip. It has a high resolution, a low cost, fast response and it is maintenance convenient and, but has a poor stability and the working condition requirements are higher. Ultrasonic detection method uses the principle of piezoelectric effect of piezoelectric materials to work. The edge position of the aluminum plate is determined based on the positive and inverse piezoelectric effect; however the detection accuracy is low.

In this paper, machine vision automatic width measurement and alignment scheme is adopted for its low cost, easy maintenance, strong adaptability to the environment, full play to the hydraulic servo system response fast, high precision, driving load capacity and other characteristics; the width measurement function is added to replace the manual width measurement and reduce the workload of workers.

\section{System Overview and Design of Centering Device}

The installation diagram of the centering device designed in this paper is shown in figure 1 .

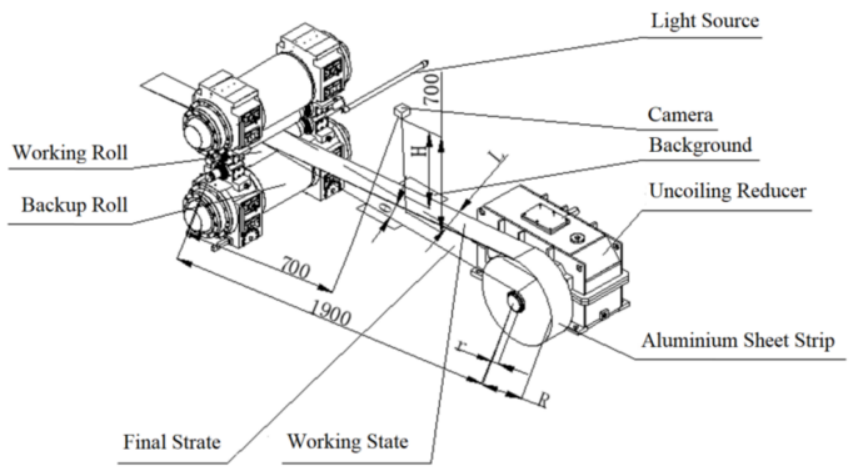

Figure 1. Schematic diagram of centering device installation

The detection camera is installed adjacent to the main control unit to realize image detection, signal input/output, data processing, communication and other functions. The actuator uses Parker DF Plus proportional valve to control the position movement of the uncoiling reducer. The light source adopts astigmatism lamp, which irradiates evenly on the aluminum strip, so that the brightness difference between the aluminum strip and the inclined section of the underside feed roller is bright. Which is convenient for noise filtering and edge detection of image information.

The position detection and deviation correction of the aluminum plate and belt of the automatic width measuring and centering equipment are completed by the machine vision [3-4]processing part and the servo control part. The image is collected by the detection camera, and the image signal is transmitted to the control center for noise filtering. The edge pixel value of the aluminum strip is obtained by using the gradient calculation method. The actual deviation and width value are obtained by converting the pixel coordinate system to the world coordinate system. The PID algorithm is used 
to obtain the voltage output signal, adjust the size of the opening of the proportional valve. And the hydraulic cylinder drives the uncoiling reducer to move and correct the position deviation. At the same time, the computer in the main operation room carries out width communication with the main control unit to obtain the strip width value (figure 2).

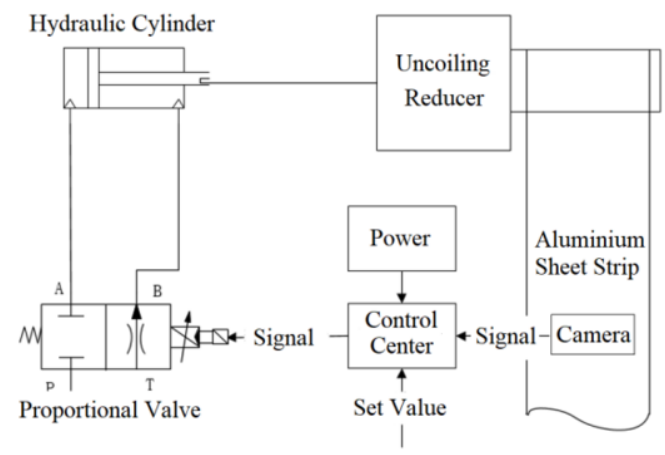

Figure 2. Connection diagram of each part of the centering control system

\section{Overview of Algorithms}

The flow of the automatic centering algorithm [5-6] is shown in figure 3 . This algorithm can realize the function of rectifying deviation and measuring width in the alignment of aluminum strip. The specific steps are as follows:

1) Calibrate the detection camera and get the internal and external parameters of the camera to measure the width of the aluminum strip.

2) Median filtering algorithm is used to filter the surface strip, block and other saltand-pepper noises of the aluminum strip.

3) After keeping good edge information, edge pixel information is obtained by using Canny edge detection algorithm; The average point of the pixel coordinate system is obtained by statistical analysis of these pixel information. The positions of pixel coordinate points obtained are converted to the world coordinate system to obtain the actual deviation value and the width value of the aluminum strip.

4) The deviation value is substituted into the PID algorithm to control the servo hydraulic valve, so as to achieve the effect of rectifying the deviation in the center; The measured width value is communicated to the computer in the main operating room via Ethernet. 


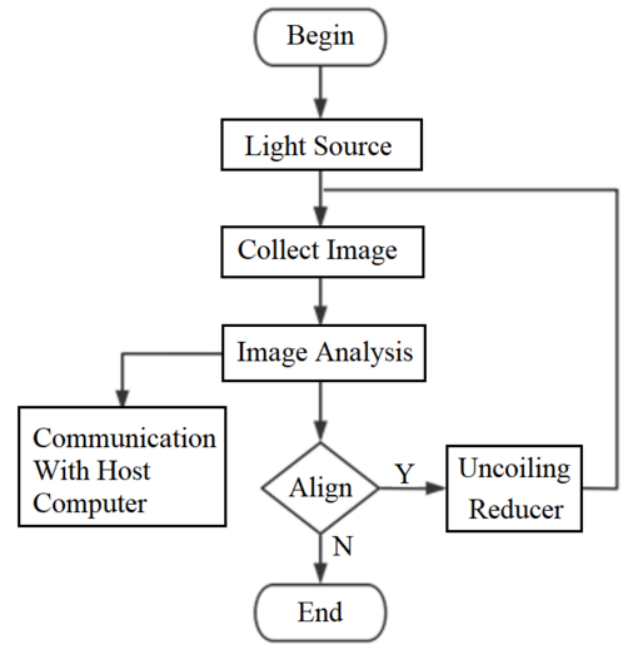

Figure 3. Algorithm flow chart

\subsection{Camera Calibration and Coordinate Conversion}

\subsubsection{Camera Calibration.}

The automatic width measuring and centering device in this paper extracts the edge information of aluminum strip with sub-pixel accuracy [7]. However, with the decrease of the roll diameter of the aluminum strip, the slope of the measured aluminum strip is always changing. The Angle between the axis of the camera lens and the normal line of the measured aluminum sheet surface is also changing. This situation will lead to perspective distortion, and then affect the accuracy of edge detection. The camera lens distortion is another major error source that affects the edge accuracy [8-9].

In order to solve the problem of perspective distortion and lens distortion, this paper uses Zhang's calibration method to calibrate the camera's internal parameters: Normalized focal length $\boldsymbol{f}_{\boldsymbol{x}}, \boldsymbol{f}_{\boldsymbol{y}}$; image main points $\boldsymbol{C}_{\boldsymbol{x}}, \boldsymbol{C}_{\boldsymbol{y}}$; radial distortion coefficients $\boldsymbol{k}_{\mathbf{1}}, \boldsymbol{k}_{\mathbf{2}}, \boldsymbol{k}_{\mathbf{3}}$; tangential distortion coefficient $\boldsymbol{p}_{\mathbf{1}}, \boldsymbol{p}_{\mathbf{2}}$, camera external parameters: rotation matrix $\boldsymbol{R}$, translation matrix $\boldsymbol{t}$.

\subsubsection{Coordinate Transformation.}

As shown in figure 4, as the roll diameter of the aluminum strip decreases, the height of the strip gradually decreases as well, however the distance between the camera and the aluminum strip gradually increases. As a result, the deviation pixels corresponding to the same deviation are different at different heights. In order to solve this problem, the detection deviations of different heights were calculated according to the geometric relationship. Secondly, the edge information detected by the camera is converted from pixel coordinates to image coordinates. Once again, the image coordinates are converted to camera coordinates, and finally the camera coordinates are converted to world coordinates to obtain the actual deviation value [10]. 


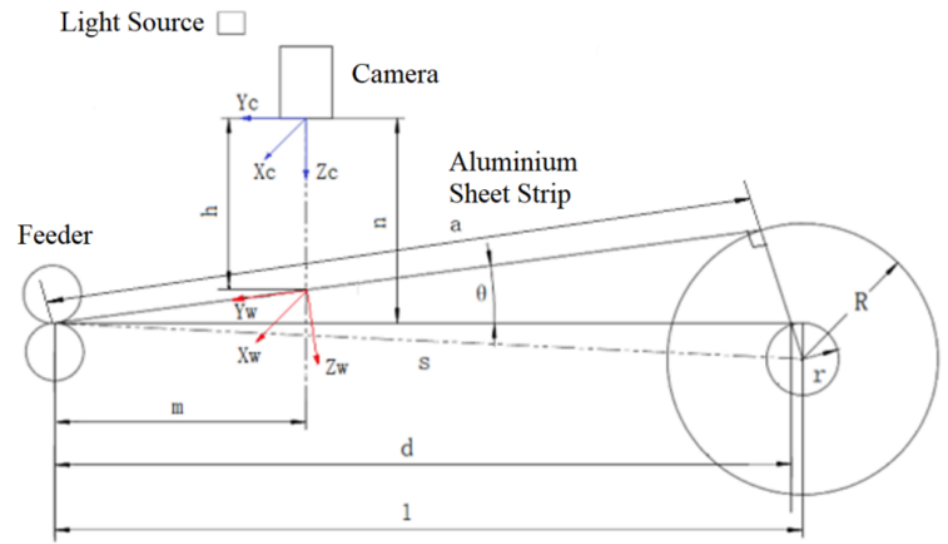

Figure 4. Schematic diagram of coordinate system transformation

According to the geometric relation in figure 4:

$$
\left\{\begin{array}{c}
R^{2}+a^{2}=r^{2}+l^{2} \\
(l-d)^{2}+r^{2}=\left(R-\sqrt{d^{2}-a^{2}}\right)^{2} \\
\tan \theta=\frac{\sqrt{d^{2}-a^{2}}}{a}
\end{array}\right.
$$

In Equation (1), $r$ is the drum diameter of the uncoiler, in $m m ; R$ is the coil diameter of the aluminum sheet, in $\mathrm{mm}$; $\mathrm{a}$ is the length of aluminum strip in $\mathrm{mm} ; \theta$ is the Angle, in degree, between the aluminum strip and the rolling process.

Equation (1) is simplified to obtain the relationship between Angle $\theta$ and the change of roll diameter $R$, as shown in Equation (2) :

$$
\tan \theta=\sqrt{\frac{\begin{array}{c}
l^{2} R^{2}-R^{4}+l^{2} r^{2}+R^{2} r^{2} \\
+2 l R r \sqrt{l^{2}+r^{2}-R^{2}}
\end{array}}{\left(R^{2}-l^{2}\right)^{2}}}
$$

In this paper, the edge information of aluminum strip and the pixel coordinate value of aluminum strip edge in pixel coordinate system can be obtained by image preprocessing and edge algorithm. The following text will introduce the relation of coordinate system transformation, and finally lead to obtain the corresponding relation between pixel coordinate points and world coordinate points.

First, the pixel coordinate is converted to the image coordinate, as shown in Equation (3), where $(u, v)$ is the pixel coordinate point, $(x, y)$ is the image coordinate point, $d_{x}$ is the width of a pixel, and $d_{y}$ is the height of a pixel.

$$
\left\{\begin{array}{l}
x=d_{x}\left(u-C_{x}\right) \\
y=d_{y}\left(v-C_{y}\right)
\end{array}\right.
$$

The image coordinates are then converted from perspective projection to camera coordinates, as shown in Equation (4), where $\left(X_{C}, Y_{C}, Z_{C}\right)$ is a point in the camera coordinate system, $f$ is the focal length of the camera, and its relationship with the normalized focal length is shown in Equation (5). 


$$
\begin{gathered}
\left\{\begin{array}{c}
X_{c}=\frac{Z_{c} x}{f} \\
Y_{c}=\frac{Z_{c} y}{f}
\end{array}\right. \\
\left\{\begin{array}{c}
f_{x}=\frac{f}{d_{x}} \\
f_{y}=\frac{f}{d_{y}} \\
Z_{c}=700-700 \tan \theta
\end{array}\right.
\end{gathered}
$$

Finally, the camera coordinates are converted through rigid body to obtain the world coordinates. See Equation (6) for the rigid body transformation relation, which consists of a rotation matrix and a translation matrix, see Equation (7), where $h=700-$ $700 \tan \theta$ is the distance between the camera coordinate system and the world coordinate system.

$$
\begin{gathered}
\left(\begin{array}{c}
X_{w} \\
Y_{w} \\
Z_{w} \\
1
\end{array}\right)=H^{-1}\left(\begin{array}{c}
X_{c} \\
Y_{c} \\
Z_{c} \\
1
\end{array}\right) \\
H=\left(\begin{array}{cc}
R & t \\
0^{T} & 1
\end{array}\right)=\left(\begin{array}{cccc}
1 & 0 & 0 & 0 \\
0 & \cos \theta & -\sin \theta & 0 \\
0 & \sin \theta & \cos \theta & h \\
0 & 0 & 0 & 1
\end{array}\right)
\end{gathered}
$$

After coordinates transformations, the actual edge deviation and strip width of aluminum strip can be obtained, as shown in Equation (8).

$$
\left(\begin{array}{c}
X_{w} \\
Y_{w} \\
Z_{w} \\
1
\end{array}\right)=\left(\begin{array}{cccc}
1 & 0 & 0 & 0 \\
0 & \cos \theta & \sin \theta & -h \sin \theta \\
0 & -\sin \theta & \cos \theta & -h \cos \theta \\
0 & 0 & 0 & 1
\end{array}\right)\left(\begin{array}{c}
\frac{Z_{c}\left(u-C_{x}\right)}{f_{x}} \\
\frac{Z_{c}\left(v-C_{y}\right)}{f_{y}} \\
Z_{c} \\
1
\end{array}\right)
$$

\subsection{Image Preprocessing and Line Detection}

After the hot rolling process of the aluminum strip, a lot of strip and block noises are often formed on the sheet surface, which have a great impact on the quality of edge detection. Therefore, it is necessary to preprocess the image captured by camera. In addition, the centering process requires image edge information, so the image details should be preserved during image noise point filtering [11].

Median filtering can effectively retain edge information and reduce the ambiguity of details while suppressing noise. The detection camera, after a frame of grayscale transformation, obtained an image $f(i, j)$, where $(i, j)$ are its pixel points. The field of $3 \times 3$ is taken to sort the 9 pixel points in the field, and the median pixel is used to 
replace $f(i, j)$ in order to achieve median filtering. The output result is shown in Equation (9), where $\mathrm{W}$ represents the rectangular region of the operation pixel, and $f(r, c)$ is the center pixel of the rectangular window.

$$
f(r, c)=\underset{(i, j)^{T} \in W}{\text { median }} \hat{f}(r-i, c-j)
$$

Canny edge operator[12] has strong anti-noise ability and can generate two information of direction and intensity at the edge, which is convenient for subsequent processing. Based on the first order differential operator, Canny edge operator adds two improvements: non-maximum suppression and double threshold. Non-maximum suppression can not only effectively suppress the multi-response edge, but also improve the positioning accuracy of the edge. The double threshold value can effectively reduce the rate of missed detection of the edge.

After Canny algorithm gets edge pixel information, Hough line detection algorithm [13] is applied to fit edge information of aluminum strip into Hough line. By analyzing the subset of points, HoughLinesP calculates the probability that these points belong to a line, which reduces the calculation amount and can effectively improve the response speed of automatic width alignment equipment.

\subsection{Servo Hydraulic Valve Pid Control}

The Parker Hannifin Corporation DF Plus proportional valve used in the automatic width-center-measuring system has an input signal range of $(-10,-1.5) \mathrm{V}$ and $(1.5,10)$ $\mathrm{V}$. There is a linear relationship between cylinder speed and voltage signal.

In the interval of $(-1.5,1.5) \mathrm{V}$, the cylinder speed is zero, that is, in the flow dead zone of the proportional valve. If only proportional control is used in the rectifying system, when the deviation is small, the proportional valve will be in the flow dead zone and the rectifying error will be too large or even lead to rectifying failure. Therefore, integral control and differential control should be added in the control process to eliminate steady-state error and improve the control performance.

The integral term of the incremental PID control algorithm [14] does not adopt cumulative errors, and the calculated results are independent of all previous accumulated errors. The incremental output signal has little influence on the stability of the system. Therefore, the incremental PID control algorithm is more suitable for the control of the automatic width measurement and centering system of the cold-rolled aluminum strip in this paper [15-16].

Let $e(t)$ be the deviation detected by the system at time $t$, the PID output $u(t)$ at this time is shown in Equation (10), where $K_{p}$ is the proportional coefficient, $K_{i}$ is the integral coefficient, $K_{d}$ is the differential coefficient, and the initial quantity is $u(0)$.

$$
u(t)=K_{p} e(t)+K_{i} \int_{0}^{t} e(t) d t+K_{d} \frac{d e(t)}{d t}+u(0)
$$

Equation (10) is discretized, integral summation is substituted by cumulative summation, and differentiation is substituted difference. Then, when the deviation of the kth is $e(k)$, PID output $u(k)$ is shown in Equation (11).

$$
u(k)=K_{p} e(k)+K_{i} \sum_{i=1}^{k} e(i)+K_{d}[e(k)-e(k-1)]+u(0)
$$

Then calculate $u(k)-u(k-1)$. The increment $\Delta u(k)$ can be obtained after simplification, as shown in Equation (12). 


$$
\begin{aligned}
u(k)= & K_{p}[e(k)-e(k-1)]+K_{i} e(k)+K_{d}[e(k)-2 e(k-1)+e(k-2)] \\
& +u(0)(12)
\end{aligned}
$$

In order to prevent disturbance caused by small error, the control dead zone should be set within the allowable error range. That is, after each deviation is obtained, it is judged whether it is in the dead zone or not. If it is not, PID control is then carried out. The flow chart is shown in figure 5 .

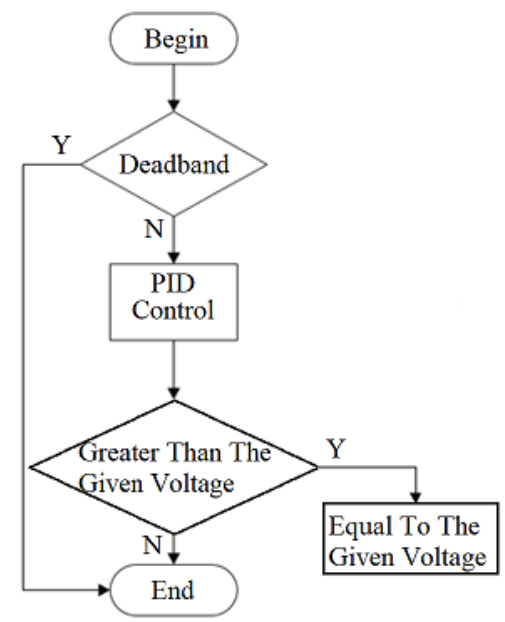

Figure 5. PID control flow chart

\section{Experimental Debugging and Application Effect}

In order to further verify the rationality of the theory, the performance of the measuring width alignment system and whether the parameters are consistent with the design, a large number of experimental data are needed. The image preprocessing and linear detection of the detection camera, the tuning of PID parameters and the overall performance of the system need experimental analysis and verification.

\subsection{Image Preprocessing and Debugging}

Through field experiments, the appropriate filtering value, the double threshold value of Canny edge detection and the input parameters of Hough line detection algorithm are debugged[17-19].

According to the comparison between figure 6 and figure 7, when the filtering value and the double threshold value of Canny edge detection are appropriate, ideal edge detection information can be obtained, but there are still interference signals, as shown in figure 7. 
(a) N-cannyleft

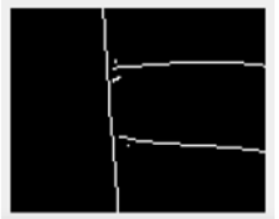

(b) N-cannyright

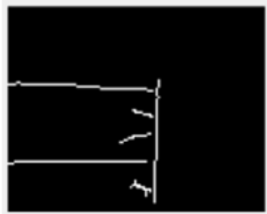

Figure 6. Edge information of aluminum strip before debugging

(a)

cannyleft

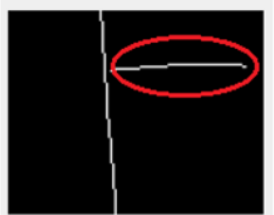

(b) cannyright

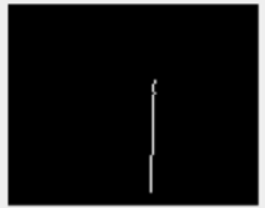

Figure 7. The first debugging edge information of aluminum strip

In view of the defects in edge detection, the edge information of aluminum strip is obtained and the pixel points are fitted in a straight line. Then, the slope of the fitting line is calculated and, obviously, the wrong lines are filtered out, so as to obtain the edge information of the aluminum strip with stable detection, as shown in figure 8 .

(a) leftline

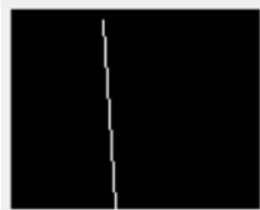

Figure 8. Final inspection information of aluminum strip

\subsection{Pid Parameter Debugging and System Performance Verification}

In the field debugging, in order to ensure the safety of equipment and avoid strip damage, the proportional link coefficient $K_{p}$ of PID system should not be too large in order to avoid system shock. Then, the steady-state error of the system is reduced by adjusting the integral coefficient $K_{i}$. The response performance of the system is improved by adjusting the differential coefficient $K_{d}$

In order to verify the performance of the system, several calibration experiments were carried out for different aluminum strips rolling process. The experiments presented in this paper are as follows: rolling speed $250-300 \mathrm{~m} / \mathrm{min}$; thickness of the strip is $4 \mathrm{~mm}$. 

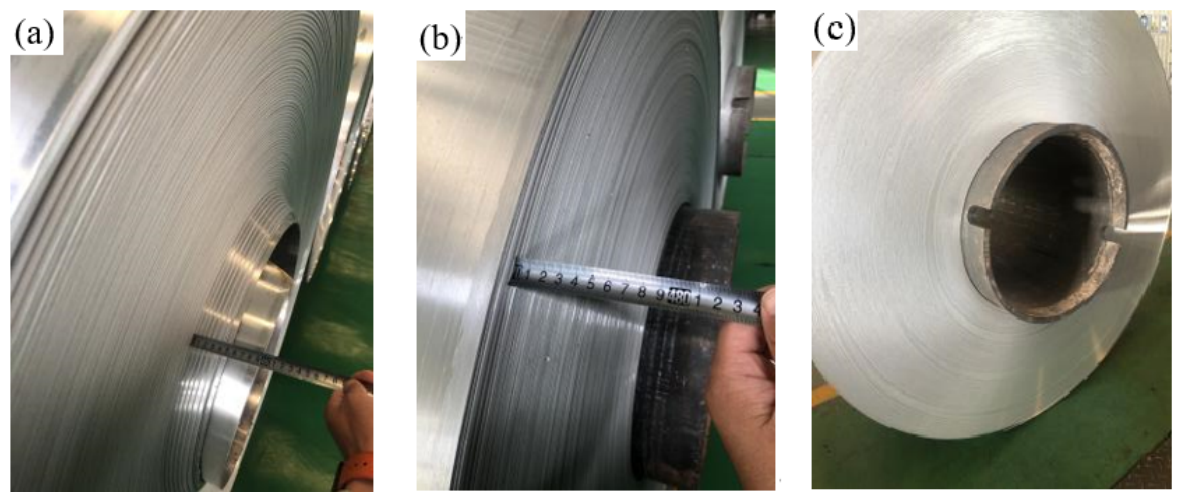

Figure 9. Edge neatness of aluminum strip

As shown in figure 9(a), the maximum deviation of the aluminum strip before rolling is about $40 \mathrm{~mm}$. Based on the principle of system stability, one adjustment cannot achieve the desired effect. The automatic width measuring and centering equipment designed in this paper can complete the centering task throughtwo-time centering correction. As shown in figure 9(b), the maximum deviation of aluminum strip after a single rolling is changed from the original $40 \mathrm{~mm}$ to $6 \mathrm{~mm}$, and the effect after another rolling is shown in figure 9(c). Which fully meets the current requirements of enterprise's aluminum strip products.

After camera calibration and conversions of coordinate systems, the width of aluminum strip measured by the automatic width alignment equipment (a) and the actual width of aluminum strip measured (b) are shown in figure 10 below. It can be seen that the width measured by the equipment meets the production requirements of the enterprise.
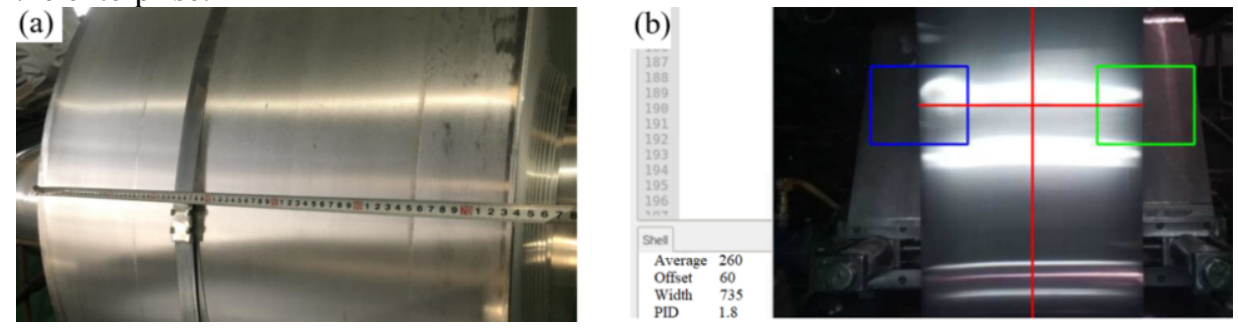

Figure 10. Width value of aluminum strip

\section{Conclusions}

Aiming at the phenomenon of aluminum strip deviation in cold rolling process, a set of automatic detection and correction scheme based on machine vision combined with the actual production is designed.

The experimental results show that the system has good stability, high degree of automation, and the centering accuracy is less than or equal to $1 \mathrm{~mm}$. Under the premise of ensuring the accuracy, the maximum rolling speed is increased from $240 \mathrm{~m} / \mathrm{min}$ to $360 \mathrm{~m} / \mathrm{min}$, and the production efficiency is significantly improved.

Automatic width measurement function instead of manual width measurement, avoid manual measurement error and reduce the workload of workers. 
The whole alignment process does not need manual participation, which avoids the influence of the operator's manual misoperation on the results, and improves the reliability of the aluminum strip conveying process.

\section{References}

[1] Song Zheng 2020 Optimization of CPC/EPC based on split layer defects in cold rolled strip. Metallurgical Autom-ation 44(04):63-69.

[2] Yao Lei 2019 Analysis of the factors in the rolling of aluminum hot-rolled coils. Aluminum Processing (01):35-37.

[3] Sun Guodong, Zhao Daxing 2015 Machine vision detection theory and algorithm. Beijing: Science Press: 35-36.

[4] Kastern Stig, Markus Ulrike, Christian Widman, Yang Shaorong, Duan Deshan, Zhang Yong, Peng Xiao, Pian Zhaohua translated 2019 Machine vision algorithm And Application 2nd Edition. Beijing: Tsinghua University Press: 347-390.

[5] Comrades, Bai Jinchi, Kang Zhiqiang 2019 Research on a new method of high-precision size measurement of linear CCD fusion grating ruler. Optical Technology 45(03): 275-281.

[6] Xu Lei, Jiang Ting, Chen Weijia 2020 Design and application of centering device and control method for aluminum alloy plate. Metallurgical Automation 44(3): 56-60.

[7] Dai Zongxian, Mo Hongbo, Zhou Yong, Zhang Zhen, Yin Aijun, Liang Zixiao 2019 Subpixel interpolation visual measurement method based on grayscale. China Testing 45(05):33-37.

[8] Zheng Dong, Feng Peng, Long Zourong, Wei Biao, Qi Wei, Yu Tingting 2020 Overview of camera calibration accuracy for multi-factor working conditions. Foreign Electronic Measurement Technology 39(08): 109-116.

[9] Jiang Meng 2020 Research on workpiece size measurement system based on monocular vision. Agricultural Equipment and Vehicle Engineering 58(01): 94-96+104.

[10] Xun Yanyan 2014 Research and realization of camera calibration technology. Inner Mongolia Agricultural University.

[11] Dai Junjie, Li Ruheng 2020 Research on edge extraction of industrial gears based on machine vision. Electronic Technology and Software Engineering (16): 127-129.

[12] Chen Shun, Meng Qingqing, Li Dengfeng 2020 Remote sensing image edge detection combined with image enhancement and improved Canny operator. Journal of Henan University (Natural Science Edition) 50(05): 623-630.

[13] Hao Zexing, Guo Gaizhi 2020 Table image correction method based on improved Canny operator and Hough transform. Journal of Inner Mongolia Normal University (Natural Science Chinese Edition) 49(05): 433-441.

[14] Wang Yichen 2018 Comparison and research of incremental PID and positional PID algorithm setting. Industrial Control Computer 31(05): 123-124.

[15] Liu Dongping 2020 Research on the design of belt conveyor belt deviation correction device. Coal Science and Technology 41(03): 110-112.

[16] Liu Zhizhong, Dong Luming 2020 Design and simulation of automatic correction device of belt conveyor based on PID control. Mechanical Engineer (06): 1-3.

[17] Liu Wei, Zhao Junfeng 2020 Design of motion detection and visual tracking platform based on Raspberry Pi. Computer Programming Skills and Maintenance (10): 140-142.

[18] Mou Xiaodong, Yang Xinfang 2020 Six steps to realize the system installation and remote control of Raspberry Pi. Newsletter (004).

[19] Mou Xiaodong, Wang Xuan 2020 Three routine camera applications of Raspberry Pi camera. Newsletter (004). 